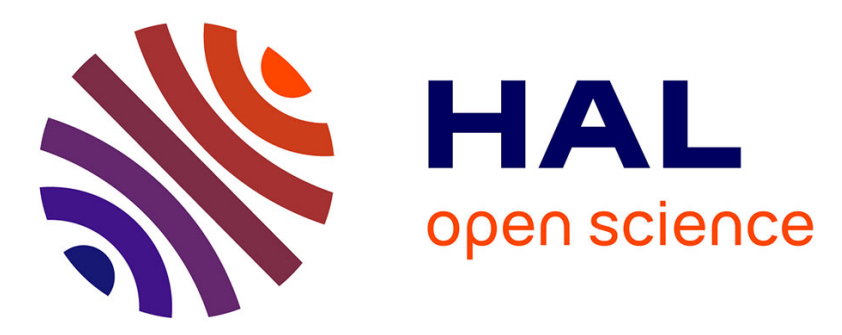

\title{
Reactivity of 3-Bromofuran in Pd-Catalyzed C-H Bond Arylation toward the Synthesis of 2,3,5-Triarylfurans
}

Arpan Sasmal, Thierry Roisnel, Jitendra Bera, Henri Doucet, Jean-François

Soulé

\section{- To cite this version:}

Arpan Sasmal, Thierry Roisnel, Jitendra Bera, Henri Doucet, Jean-François Soulé. Reactivity of 3Bromofuran in Pd-Catalyzed C-H Bond Arylation toward the Synthesis of 2,3,5-Triarylfurans. Synthesis: Journal of Synthetic Organic Chemistry, 2019, 51 (17), pp.3241-3249. 10.1055/s-0037-1611819 . hal-02130076

\section{HAL Id: hal-02130076 https://hal.science/hal-02130076}

Submitted on 26 Nov 2020

HAL is a multi-disciplinary open access archive for the deposit and dissemination of scientific research documents, whether they are published or not. The documents may come from teaching and research institutions in France or abroad, or from public or private research centers.
L'archive ouverte pluridisciplinaire HAL, est destinée au dépôt et à la diffusion de documents scientifiques de niveau recherche, publiés ou non, émanant des établissements d'enseignement et de recherche français ou étrangers, des laboratoires publics ou privés. 


\title{
Reactivity of 3-Bromofuran in Pd-Catalyzed C-H Bond Arylation Toward the Synthesis of 2,3,5-Triaryl Furans
}

\author{
Arpan Sasmal ${ }^{\mathrm{a}}$ \\ Thierry Roisnel ${ }^{\mathrm{a}}$ \\ Jitendra K. Bera ${ }^{b}$ \\ Henri Doucet*a \\ Jean-François Soulé*a \\ a Univ Rennes, CNRS, ISCR UMR 6226,F-35000 Rennes, France. \\ ${ }^{\mathrm{b}}$ Department of Chemistry and Center for Environmental \\ Science and Engineering, Indian Institute of Technology \\ Kanpur, Kanpur 208016, India. \\ *Henri.doucet@univ-rennes1.fr \& jean-francois.soule@univ- \\ rennes1.fr
}

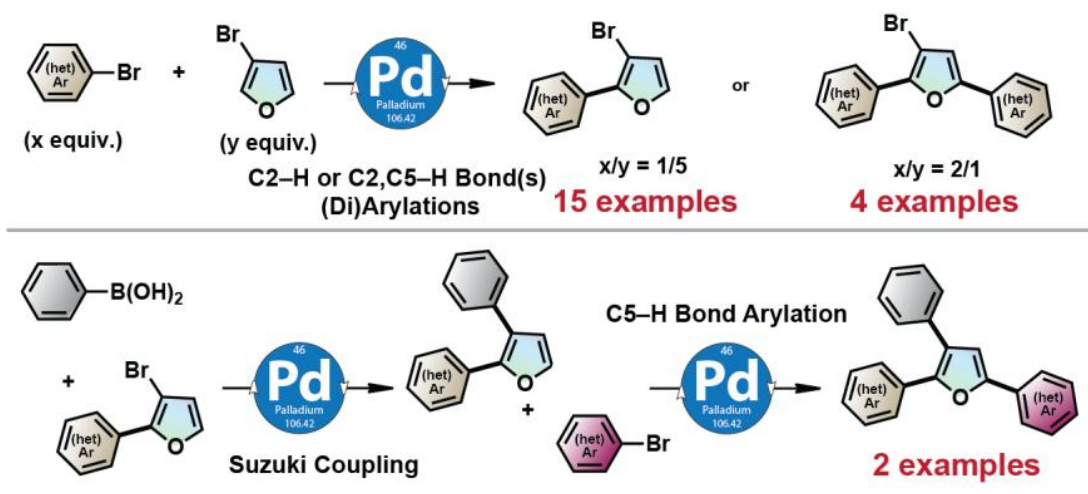

Abstract The $\mathrm{C}-\mathrm{H}$ bond arylation at $\mathrm{C} 2$ position of 3-bromofuran was performed using aryl bromides as coupling partners in the presence of phosphine free $\mathrm{Pd}(\mathrm{OAc})_{2} / \mathrm{KOAc}$ in DMA. C2,C5-di- or even $\mathrm{C2}, \mathrm{C} 4, \mathrm{C} 5$-tri-arylated 3bromofuran derivatives were obtained using a larger amount of aryl bromides. Then, C2,C3,C5-triarylated furans - containing three different aryl groups- were synthesized via $\mathrm{C} 2-\mathrm{H}$ bond arylation, Suzuki reaction, and $\mathrm{C} 5-\mathrm{H}$ bond arylation sequence.

Key words Palladium, Furans, C-H Bond Arylation, Aryl Bromides

Substituted furans are omnipresent in natural products, ${ }^{1}$ pharmaceuticals, ${ }^{2}$ (bio-sourced) polymeric materials, ${ }^{3}$ and other functional molecules. ${ }^{4}$ They are also useful synthetic intermediates. ${ }^{5}$ Arylated furans are important frameworks embedded in many important pharmaceuticals drugs. As examples, Azimilide ${ }^{\circledR}$ is a class III antiarrhythmic drug, Neurodazine ${ }^{\circledR}$ affects neuronal cell differentiation and Lapatinib ${ }^{\circledR}$ is an orally active drug for breast cancer and other solid tumours.

Figure 1. Relevant Drugs Containing a 2-Arylfuran Motif
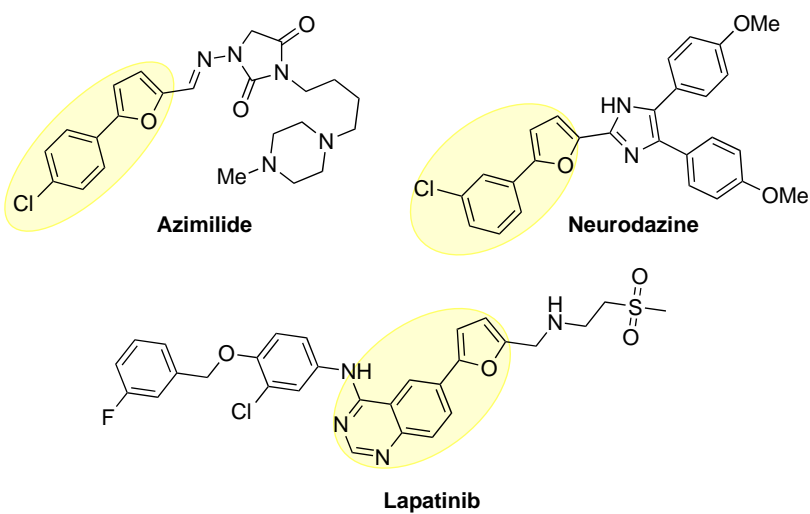

Therefore, the development of efficient and regioselective synthetic pathways of multi-substituted furans has been among important research subjects in heterocyclic chemistry. Beside the traditional approaches involving cyclocondensation, cycloisomerization or multi-steps synthesis, ${ }^{6}$ the direct functionalization of 
furan rings via palladium catalyzed $\mathrm{C}-\mathrm{H}$ bond arylation is a modular approach to access to polyarylated furans. ${ }^{7}$ The first example of Pd-catalyzed $\mathrm{C} 2-\mathrm{H}$ bond arylation of furan with activated aryl bromides was reported in 1990 by Ohta and co-workers using $5 \mathrm{~mol} \% \mathrm{Pd}\left(\mathrm{PPh}_{3}\right)_{4}$ associated with KOAc in DMA at 150 ${ }^{\circ} \mathrm{C}$ (Figure 2a). ${ }^{8}$ However, low to moderate yields in favor of the $\mathrm{C} 2$-arylated furans were often obtained due to the high reactivity of $\mathrm{C} 5-\mathrm{H}$ bond of $\mathrm{C} 2$-arylated furans leading to the formation of $\mathrm{C} 2$, $\mathrm{C} 5$-diarylated furans. ${ }^{9}$ On the other hand, the reactivity of C3-substituted furans attracted less attention. The regioselectivity control of the $\mathrm{C}-\mathrm{H}$ bond arylation of ethyl 3-furoate depends on the reaction conditions. In 2003, Sharp and co-workers reported the C2 arylation with electron-deficient aryl bromides using 5 mol\% $\mathrm{Pd}\left(\mathrm{PPh}_{3}\right)_{4}$ associated with KOAc in non-polar solvents such as toluene at $110{ }^{\circ} \mathrm{C}$ (Figure $2 \mathrm{~b}$, right). ${ }^{10}$ In contrast, they reported two examples of C5-arylation of ethyl 3-furoate using polar solvents, such as NMP, with Pd/C as catalyst (Figure 2b, left). ${ }^{10}$ Later, Doucet and co-workers also investigated the regioselectivity of $\mathrm{C}-\mathrm{H}$ bond arylation of ethyl 3 -furoate and observed that using $0.2 \mathrm{~mol} \%$ $\mathrm{Pd}(\mathrm{OAc})_{2} / \mathrm{dppb}$ in the presence of KOAc in DMA, C5-arylated furans were obtained as major products along with the formation of C2-arylated furans as minor products (Figure 2c).11 Doucet and co-worker also reported one example of C2-arylation of 3-formylfuran using a similar catalytic system (Figure $2 \mathrm{~d}$ ). ${ }^{12}$ Conversely, the chemoselective $\mathrm{C}-\mathrm{H}$ bond arylations of thiophenes or pyrazoles bromo-substituted at $\mathrm{C} 3$ or C4 positions with aryl halides allowed the construction of polyarylated heteroaromatics. ${ }^{13}$ To the best of our knowledge, the reactivity of 3-bromofuran in direct arylation was not reported yet. Herein, we investigate on the coupling of 3-bromofuran with electron-deficient aryl bromides and on their polyarylation to construct triarylated furans (Figure 2e).

Figure 2. Reactivity of (Substituted) Furan Derivatives in Pd-Catalyzed C-H Bond Arylation

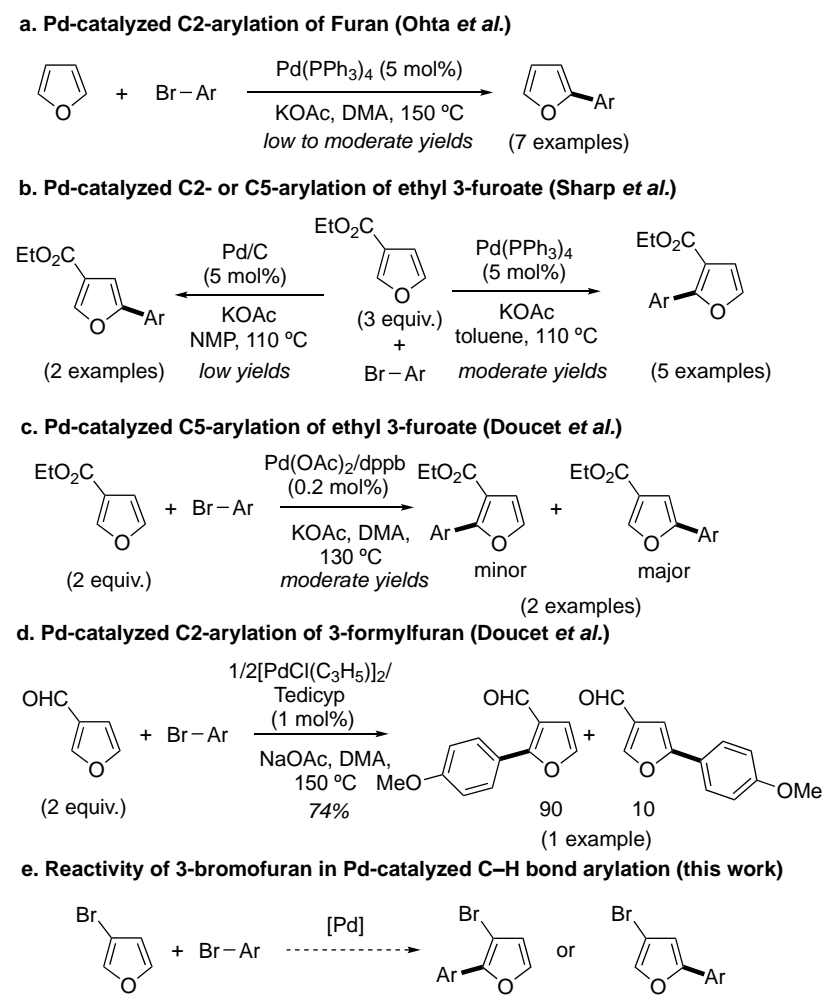

We selected 4-bromoacetophenone as the coupling partner to investigate the chemoselectivity of the coupling with 3-bromofuran. During the reaction development, we determined that the best conditions to regioselectively obtain C2-arylated 3-bromofuran 1 in $47 \%$ yield were $1 \mathrm{~mol} \%$ of $\mathrm{Pd}(\mathrm{OAc})_{2}$ as catalyst, 2 equivalents of KOAc as base in DMA at $120{ }^{\circ} \mathrm{C}$ over $8 \mathrm{~h}$ (Table 1 , entry 1). The regioselectivity of the 
arylation at C2 position was confirmed by X-ray-analysis of 1.14,15 The amount of 3-bromofuran (5 equivalents) was critical to isolate $\mathbf{1}$ in a good yield due to oligomerization as major side reaction (Table 1, entries 1 and 2). The reaction time was also a very important factor for this coupling, as shorter ( $4 \mathrm{~h})$ or longer reaction times $(16 \mathrm{~h}$ ) resulted in lower yields (Table 1, entries 3 and 4 ). A higher temperature of $150{ }^{\circ} \mathrm{C}$ led to oligomerization, while at $80{ }^{\circ} \mathrm{C}$ a partial conversion of 4 -bromoacetophenone was observed (Table 1, entries 5 and 6). Other palladium sources than $\mathrm{Pd}(\mathrm{OAc})_{2}$ as well as different catalyst loadings gave lower yields in $\mathbf{1}$ (Table 1, entries 7-11). Reactions performed in other solvents such as cyclopentyl methyl ether (CPME), 1,4-dioxane or pentan-1-ol failed to afford the arylated furan $\mathbf{1}$ (Table 1 , entries 12-14). The concentration plays a major role to avoid the oligomerization. Indeed, 0.125 molar was determined to be the best choice, as a concentration of 0.25 molar afforded more oligomers and when the reaction was performed at 0.1 molar a partial conversion was obtained (Table 1, entries 16 and 17). Finally, our attempts to inhibit the degradation of 3-bromofuran by addition of TEMPO or catechol were unsuccessful (Table 1, entries 17 and 18).

Table 1. Reaction Development

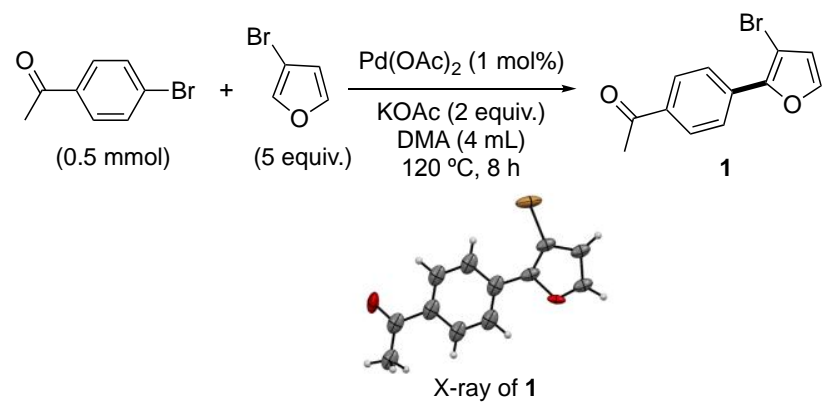

\begin{tabular}{lll}
\hline Entry & Variation from standard conditions & Yield in $\mathbf{1}(\%)^{[a]}$ \\
\hline 1 & - & $52(47)$ \\
2 & 3 equiv. of 3-bromofuran & 26 \\
3 & $4 \mathrm{~h}$ & 32 \\
4 & $16 \mathrm{~h}$ & 49 \\
5 & $150 \stackrel{\circ}{ } \mathrm{C}$ & traces \\
6 & $80{ }^{\circ} \mathrm{C}$ & 22 \\
7 & $\mathrm{PdCl}_{2}(1 \mathrm{~mol} \%)$ & 48 \\
8 & $\mathrm{PdCl}_{2}\left(\mathrm{CH}_{3} \mathrm{CN}\right)_{2}(1$ mol\%) & 42 \\
9 & $\mathrm{PdCl}\left(\mathrm{C}_{3} \mathrm{H}_{5}\right)(\mathrm{dppb})(1 \mathrm{~mol} \%)$ & 25 \\
10 & $\mathrm{Pd}(\mathrm{OAc})_{2}(2$ mol\%) & 49 \\
11 & $\mathrm{Pd}(\mathrm{OAc})_{2}(0.5 \mathrm{~mol} \%)$ & 42 \\
12 & $\mathrm{cyclopentyl} \mathrm{methyl} \mathrm{ether}(\mathrm{CPME})$ & traces \\
13 & $1,4-d i o x a n e$ & traces \\
14 & $\mathrm{Pentan}-1-\mathrm{ol}$ & traces \\
15 & $\mathrm{DMA}(2 \mathrm{~mL})$ & 29 \\
16 & $\mathrm{DMA}(5 \mathrm{~mL})$ & 42 \\
17 & $\mathrm{TEMPO}(0.2$ equiv.) as additive & 45 \\
28 & Catechol $(0.2$ equiv.) as additive & 41 \\
\hline
\end{tabular}

[a] determined by GC-MS analysis using $n$-dodecane as

internal standard. Isolated yield is shown in parentheses

Having determined the best conditions to selectively arylate the $\mathrm{C}-\mathrm{H}$ bond at $\mathrm{C} 2$ position of 3-bromofuran without cleavage of the $\mathrm{C}-\mathrm{Br}$ bond on the furan ring, we turned our attention to the scope of aryl 
bromides (Scheme 1). Firstly, the reactivity of a set of para-substituted aryl bromides was investigated. The reaction proceeded smoothly using aryl bromides bearing an electron-withdrawing group such as cyano, trifluoromethyl, propionyl, or chloro affording the C2-arylated 3-bromofurans $\mathbf{2 - 5}$ in moderate yields. Notably, when non-activated aryl bromides were employed (e.g., bromobenzene or 4bromoanisole), the corresponding C2-arylated 3-bromofurans were not obtained due to the oligomerization of 3-bromofuran. Meta-substituted aryl bromides, such as 3-bromobenzonitrile and 3bromobenzotrifluoride were chemoselectively coupled with 3-bromofuran to give the arylated compounds 6 and $\mathbf{7}$ in 50\% yields, again without formation of diarylated products. The reaction was slightly sensitive to steric hindrance, as from 2-bromobenzonitrile the coupling product $\mathbf{8}$ was isolated in only $42 \%$ yield. From less bulky and activated 4-bromo-2-fluorobenzaldehyde, Pd-catalyzed C2 arylation lead to the furan derivative 9 in 52\% yield. 2-Bromonaphtalene displayed a higher reactivity than 1bromonaphtalene in Pd-catalyzed C2 arylation of 3-bromofuran, owing steric factors. 3-Bromopyridine was also successfully coupled with 3-bromofuran to afford $\mathbf{1 2}$ in good yield. Other $\mathrm{N}$-heteroaryl bromides such as 5-bromopyrimidine and 3-bromoquinoline gave the C2-heteroarylated 3-bromofuran derivatives 13 and 14 in $45 \%$ and 50\% yield, respectively.

Scheme 1. Scope of (Hetero)Aryl Bromides in Pd-Catalyzed Direct C2-Arylation of 3-Bromofuran

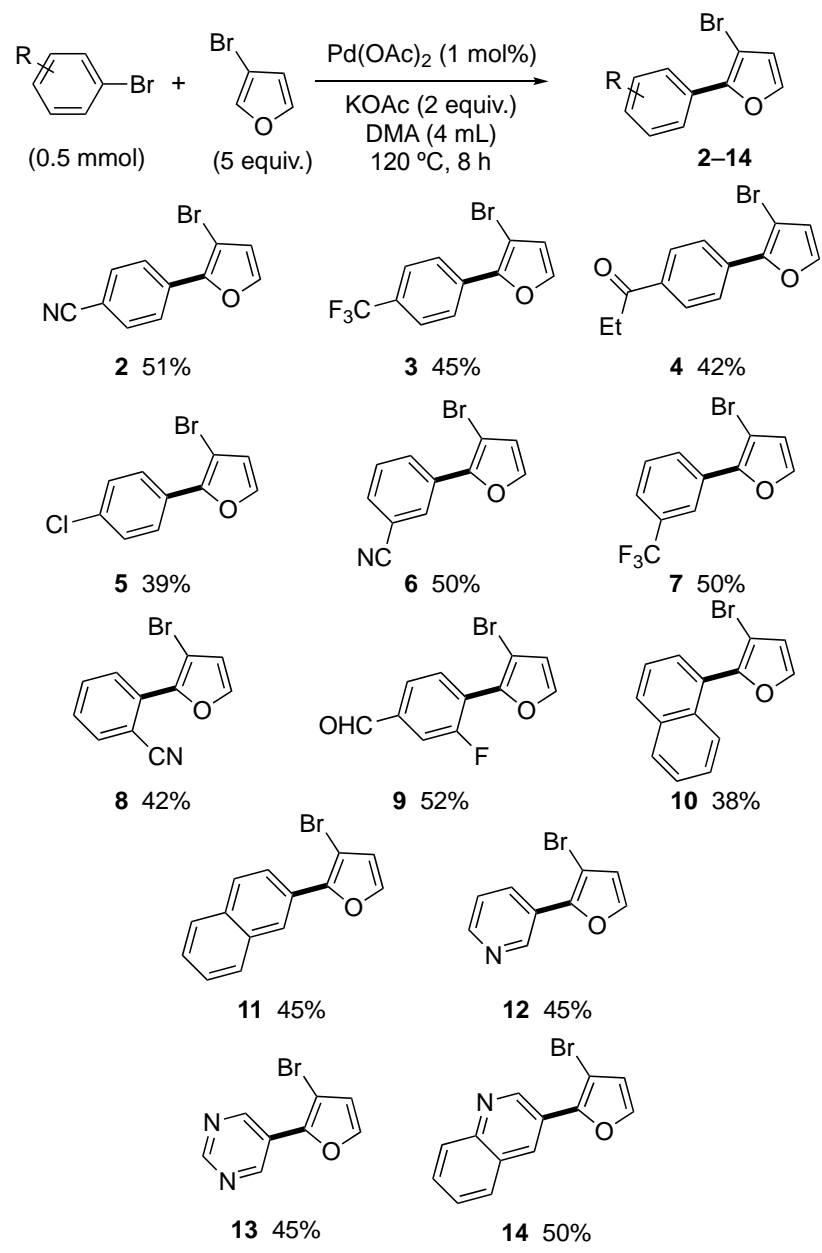

$\mathrm{C}-\mathrm{H}$ bond arylation of furan using palladium catalysis often occurred at $\mathrm{C} 2$ and $\mathrm{C} 5$ positions. ${ }^{8}$ We then decided to employ an excess amount of aryl bromides to have access to C2,C5-diarylated 3-bromofurans in one step (Scheme 2). Reaction from 2 equivalents of 4-bromobenzonitrile and 3-bromofuran in the presence of $2 \mathrm{~mol} \% \mathrm{Pd}(\mathrm{OAc})_{2}$ associated with 3 equivalents of KOAc did not afford the desired diarylated product 15, but only mono-arylated furan 2 albeit in a poor yield. Surprisingly, in the presence of 2 equivalents of 4-bromobenzotrifluoride, 3-bromofuran was diarylated at C2 and C5 positions to give the 
furan 16 in 56\% yield. This reactivity was also observed with 2-or 3-bromobenzotrifluorides and 2bromo-6-(trifluoromethyl)pyridine, as the diarylated furans 17-19 were selectively obtained in 47-53\% yields. It is noteworthy to mention that in all cases, the formation of mono-arylated 3-bromofurans was also observed in 5-10\% yields. The reason of the outperformance of aryl bromides bearing a trifluoromethyl group for the multi $\mathrm{C}-\mathrm{H}$ bond arylations remains unclear. However, similar phenomena have been previously observed in the Pd-catalyzed multiple $\mathrm{C}-\mathrm{H}$ bond arylations of other heterocycles (e.g., thiophenes, pyrroles, imidazoles and benzofuran). ${ }^{16}$

Scheme 2. Scope of Hetero)Aryl Bromides in Pd-Catalyzed Direct C2,C5-Diarylation of 3-Bromofuran
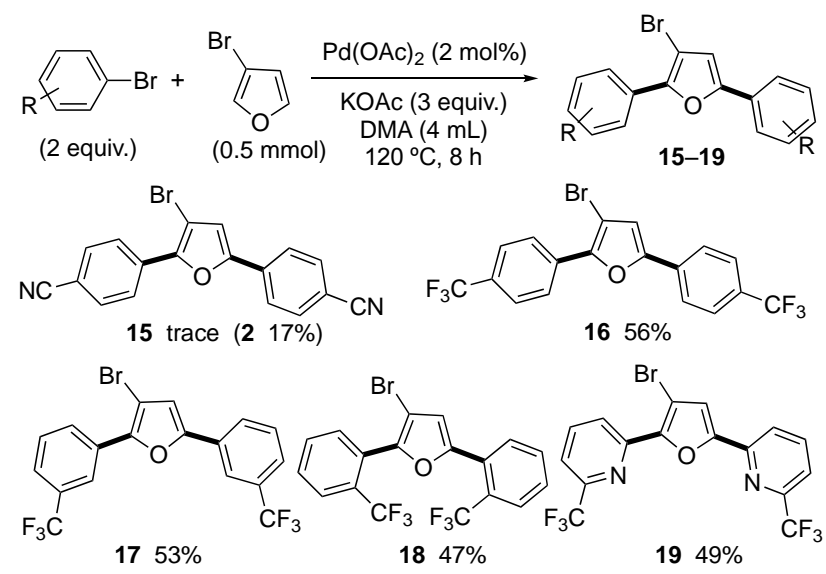

We also performed the multiple $\mathrm{C}-\mathrm{H}$ bond arylation of 3-bromofuran with 1-bromo-3,5bis(trifluoromethyl)benzene using $2 \mathrm{~mol} \%$ of $\mathrm{Pd}(\mathrm{OAc})_{2}$ as catalyst in DMA (Scheme 3). In the presence of 2 equivalents of this aryl bromide with 3 equivalents of base, the formation of triarylated furan $\mathbf{2 0}$ as well as the formation of a diarylated furan was observed. Notably, we were not able to separate them by flash chromatography. Furthermore, we set-up the reaction using 4 equivalents of 1-bromo-3,5bis(trifluoromethyl)benzene to get selectively the triarylated furan 20 in good $61 \%$ yield.

Scheme 3. Pd-catalyzed Direct C2,C4,C5-Triarylation of 3-Bromofuran with 1-Bromo-3,5-

bis(trifluoromethyl)benzene

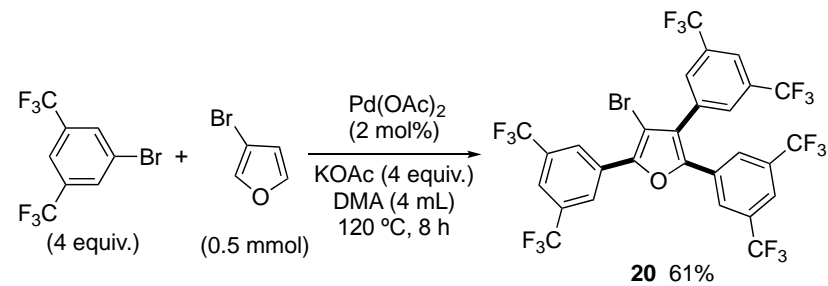

We also tried to prepare 2,5-diarylated 3-bromofurans with two different aryl units by iterative C-H bond arylations (Scheme 4). However, our attempts to selectively arylate the $\mathrm{C} 5-\mathrm{H}$ bond of 3-bromo-2(naphthalen-1-yl)furan (10) with 4-bromobenzonitrile remained unsuccessful even in the presence of the highly active diphosphine-palladium complex $\left[\mathrm{PdCl}\left(\mathrm{C}_{3} \mathrm{H}_{5}\right)(\mathrm{dppb})\right]$. Instead, a complex mixture of side products was observed by GC-MS analysis, which included debrominated products.

Scheme 4. Reactivity of 2-Naphtyl-3-bromofuran in Pd-Catalyzed Direct C5-Arylation 


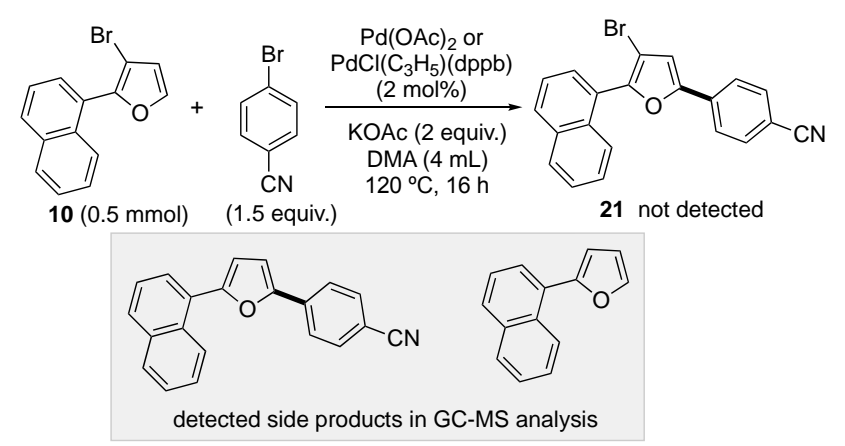

Therefore, we decided to transform the $\mathrm{C}-\mathrm{Br}$ bond into a less reactive $\mathrm{C}-\mathrm{Ph}$ bond to later investigate the reactivity of the $\mathrm{C} 5-\mathrm{H}$ bond. From a mixture of 3-bromo-2-(naphthalen-1-yl)furan (10) and phenylboronic acid (1.5 equivalents) in the presence of $2 \mathrm{~mol} \%$ of a diphosphine-palladium catalyst and 2 equivalents of $\mathrm{K}_{3} \mathrm{PO}_{4}$ in dioxane at $100{ }^{\circ} \mathrm{C}$ over $16 \mathrm{~h}$, the 2,3-diarylfuran 22 was obtained in $78 \%$ yield (Scheme 5).

Scheme 5. Reactivity of C-Br Bond of 2-Naphtyl-3-Bromofuran in Pd-Catalyzed Suzuki Reaction

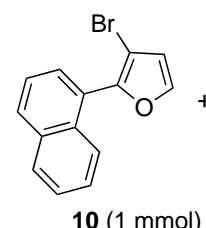

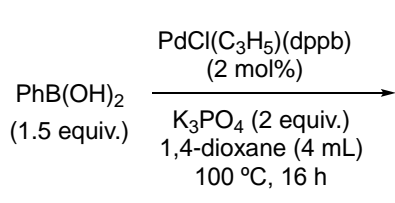

$100^{\circ} \mathrm{C}, 16 \mathrm{~h}$

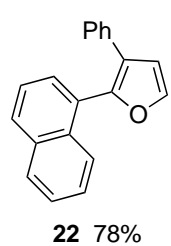

$2278 \%$

Only few methods are reported to prepare 2,3,5-triaryl furans containing three different aryl groups and they involve cyclization reactions. ${ }^{17}$ To the best of our knowledge, there is no example of direct arylation of 2,3-diarylfurans. Therefore, we further demonstrated the potential of this methodology with the introduction of a third aryl group, by the sequential synthesis of 2,3,5-triarylfuran derivatives containing three different aryl units (Scheme 6). From a mixture of 2-naphtyl-3-phenylfuran (22) and 4bromobenzonitrile (1.5 equiv.) in the presence of $\mathrm{PdCl}\left(\mathrm{C}_{3} \mathrm{H}_{5}\right)(\mathrm{dppb})(2 \mathrm{~mol} \%)$ associated with KOAc as base in DMA at $150{ }^{\circ} \mathrm{C}$, the arylated product 23 was obtained in $71 \%$ yield. The arylation took place at the most acidic $\mathrm{C}-\mathrm{H}$ bond, namely $\mathrm{C} 5$ position. Similarly, the reaction with 3-bromoquinoline afforded the 2,3,5-triaryl furan 24 in $81 \%$ yield.

Scheme 6. Reactivity of 2-Naphtyl-3-phenylfuran in Pd-Catalyzed Direct C5-Arylation
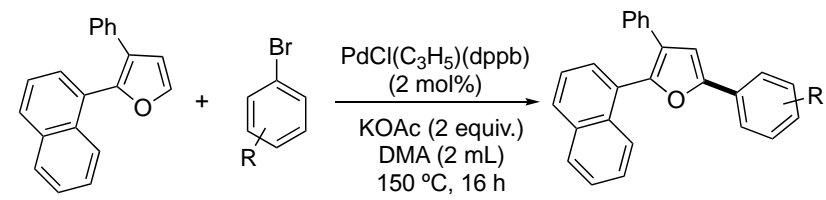

$22(0.5 \mathrm{mmol}) \quad$ ( 1.5 equiv. $)$

23 and 24

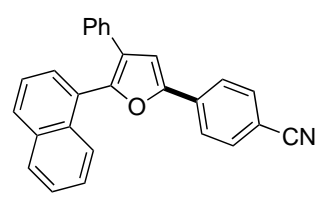

$2371 \%$

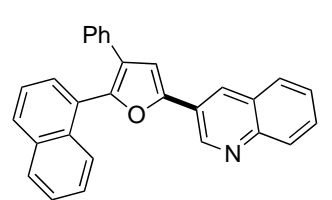

$2481 \%$

In summary, we have demonstrated that 3-bromofuran is an attractive platform for the synthesis of polyarylated furans. Firstly, conditions for the regioselective $\mathrm{C} 2-\mathrm{H}$ bond arylation of 3-bromofuran with activated aryl bromides have been developed using phosphine-free $\mathrm{Pd}(\mathrm{OAc})_{2}$ catalyst. Interestingly, the 
reaction was very chemoselective. There was no formation of products resulting from the activation of the $\mathrm{C}-\mathrm{Br}$ bond on the furan ring and this $\mathrm{C}-\mathrm{Br}$ bond remained untouched after the $\mathrm{C} 2$-arylation if the aryl bromide bears an electron-withdrawing group. The use of a larger amount of aryl bromide couplings partners allowed the access to C2,C5-diarylfurans and even a C2,C4,C5-triarylfuran in the case of 3,5bis(trifluoromethyl)bromobenzene. Moreover, C2,C3,C5-triarylfurans containing three different aryl units have been prepared in good yields via $\mathrm{C} 2-\mathrm{H}$ bond arylation, Suzuki cross-coupling reaction, and C5$\mathrm{H}$ bond arylation sequence. 


\section{Experimental Section}

All reactions were carried out under argon atmosphere with standard Schlenk techniques. DMA was purchased from Acros Organics and was not purified before use. ${ }^{1} \mathrm{H}$ and ${ }^{13} \mathrm{C}$ NMR spectra are recorded on Bruker AV III $400 \mathrm{MHz}$ NMR spectrometer equipped with BBFO probehead. Chemical shifts $(\delta)$ are reported in parts per million relative to residual chloroform (7.28 ppm for ${ }^{1} \mathrm{H} ; 77.23 \mathrm{ppm}$ for ${ }^{13} \mathrm{C}$ ), constants are reported in Hertz. ${ }^{1} \mathrm{H}$ NMR assignment abbreviations are the following: singlet (s), doublet $(d)$, triplet $(\mathrm{t})$, quartet $(\mathrm{q})$, doublet of doublets $(\mathrm{dd})$, doublet of triplets (dt), and multiplet $(\mathrm{m})$. All reagents were weighed and handled in air.

\section{Procedures}

General procedure for the C2-arylation reaction of 3-bromofuran: As a typical experiment, the reaction of the aryl bromide ( $0.5 \mathrm{mmol}), 3$-bromofuran $(2.5 \mathrm{mmol}, 5$ equiv., $367 \mathrm{mg}$ ) and KOAc $(1 \mathrm{mmol}, 2$ equiv., $98 \mathrm{mg}$ ) at $120^{\circ} \mathrm{C}$ during $8 \mathrm{~h}$ in DMA ( $2 \mathrm{~mL}$ ) in the presence of $\mathrm{Pd}(\mathrm{OAc})_{2}(1 \mathrm{~mol} \%, 0.005 \mathrm{mmol}, 1.12$ $\mathrm{mg}$ ) (see tables or schemes) under argon affords the arylation product after evaporation of the solvent and purification on silica gel.

1-(4-(3-Bromofuran-2-yl)phenyl)ethan-1-one 1 : From 4-bromoacetophenone (100 mg, $0.5 \mathrm{mmol}$ ) and 3-bromofuran ( $2.5 \mathrm{mmol}, 5$ equiv., $367 \mathrm{mg}$ ), 1 was obtained in $47 \%$ (62 mg) yield.

${ }^{1} \mathrm{H} \mathrm{NMR}\left(400 \mathrm{MHz}, \mathrm{CDCl}_{3}, 25^{\circ} \mathrm{C}\right): \delta(\mathrm{ppm})=8.11(\mathrm{~d}, J=8.7 \mathrm{~Hz}, 2 \mathrm{H}), 8.04(\mathrm{~d}, J=8.7 \mathrm{~Hz}, 2 \mathrm{H}), 7.50(\mathrm{~d}, J=2.0$ $\mathrm{Hz}, 1 \mathrm{H}), 6.61(\mathrm{~d}, J=2.0 \mathrm{~Hz}, 1 \mathrm{H}), 2.65(\mathrm{~s}, 3 \mathrm{H})$.

${ }^{13} \mathrm{C}$ NMR $\left(100 \mathrm{MHz}, \mathrm{CDCl}_{3}, 2{ }^{\circ} \mathrm{C}\right): \delta(\mathrm{ppm})=197.6,148.0,142.9,136.1,134.0,128.8,125.3,117.0,98.5$, 26.8 .

Elemental analysis: calcd (\%) for $\mathrm{C}_{12} \mathrm{H}_{9} \mathrm{BrO}_{2}$ (265.11): C 54.37, H 3.42; found: C 54.59, H 3.21.

4-(3-Bromofuran-2-yl)benzonitrile 2: From 4-bromobenzonitrile (91 mg, $0.5 \mathrm{mmol}$ ) and 3-bromofuran (367 mg, $2.5 \mathrm{mmol}, 5$ equiv.), 2 was obtained in 51\% (63 mg) yield.

${ }^{1} \mathrm{H} \mathrm{NMR}\left(400 \mathrm{MHz}, \mathrm{CDCl}_{3}, 2{ }^{\circ} \mathrm{C}\right): \delta(\mathrm{ppm})=8.09(\mathrm{~d}, J=8.4 \mathrm{~Hz}, 2 \mathrm{H}), 7.69(\mathrm{~d}, J=8.4 \mathrm{~Hz}, 2 \mathrm{H}), 7.49(\mathrm{~d}, J=1.7$ $\mathrm{Hz}, 1 \mathrm{H}), 6.59$ (d, $J=1.7 \mathrm{~Hz}, 1 \mathrm{H})$.

${ }^{13} \mathrm{C}$ NMR $\left(100 \mathrm{MHz}, \mathrm{CDCl}_{3}, 2{ }^{\circ} \mathrm{C}\right): \delta(\mathrm{ppm})=147.0,143.3,133.8,132.5,125.6,118.9,117.1,111.2,99.3$.

Elemental analysis: calcd (\%) for $\mathrm{C}_{11} \mathrm{H}_{6} \mathrm{BrNO}$ (248.08): C 53.26, H 2.44; found: C 53.09, H 2.23.

3-Bromo-2-(4-(trifluoromethyl)phenyl)furan 3: From 4-bromobenzotrifluoride (113 mg, $0.5 \mathrm{mmol}$ ) and 3-bromofuran (367 mg, $2.5 \mathrm{mmol}, 5$ equiv.), 3 was obtained in 45\% (65 mg) yield.

$1 \mathrm{H} \mathrm{NMR}\left(400 \mathrm{MHz}, \mathrm{CDCl}_{3}, 25^{\circ} \mathrm{C}\right): \delta(\mathrm{ppm})=8.12(\mathrm{~d}, J=8.4 \mathrm{~Hz}, 2 \mathrm{H}), 7.70(\mathrm{~d}, J=8.4 \mathrm{~Hz}, 2 \mathrm{H}), 7.50(\mathrm{~d}, J=1.9$ $\mathrm{Hz}, 1 \mathrm{H}), 6.61(\mathrm{~d}, J=1.9 \mathrm{~Hz}, 1 \mathrm{H})$.

${ }^{13} \mathrm{C}$ NMR $\left(100 \mathrm{MHz}, \mathrm{CDCl}_{3}, 25^{\circ} \mathrm{C}\right): \delta(\mathrm{ppm})=147.5,142.6,132.9,128.4,125.5(\mathrm{q}, J=4.1 \mathrm{~Hz}), 125.4,124.0$ (q, $J=274.2 \mathrm{~Hz}), 116.6,98.0$.

19F NMR (376 MHz, $\left.\mathrm{CDCl}_{3}, 25^{\circ} \mathrm{C}\right): \delta(\mathrm{ppm})=-62.7(\mathrm{~s})$.

Elemental analysis: calcd (\%) for $\mathrm{C}_{11} \mathrm{H}_{6} \mathrm{BrF}_{3} \mathrm{O}$ (291.07): C 45.39, H 2.08; found: C 45.51, H 2.34.

1-(4-(3-Bromofuran-2-yl)phenyl)propan-1-one 4: From 4-bromopropiophenone (107 mg, $0.5 \mathrm{mmol}$ ) and 3-bromofuran (367 mg, $2.5 \mathrm{mmol}, 5$ equiv.), 4 was obtained in 42\% (59 mg) yield.

${ }^{1} \mathrm{H} \mathrm{NMR}\left(400 \mathrm{MHz}, \mathrm{CDCl}_{3}, 25^{\circ} \mathrm{C}\right): \delta(\mathrm{ppm})=8.08(\mathrm{~d}, J=8.8 \mathrm{~Hz}, 2 \mathrm{H}), 8.02(\mathrm{~d}, J=8.9 \mathrm{~Hz}, 2 \mathrm{H}), 7.47(\mathrm{~d}, J=1.9$ $\mathrm{Hz}, 1 \mathrm{H}), 6.58(\mathrm{~d}, J=1.9 \mathrm{~Hz}, 1 \mathrm{H}), 3.02(\mathrm{q}, J=7.2 \mathrm{~Hz}, 2 \mathrm{H}), 1.25(\mathrm{t}, J=7.2 \mathrm{~Hz}, 3 \mathrm{H})$. 
${ }^{13} \mathrm{C}$ NMR $\left(100 \mathrm{MHz}, \mathrm{CDCl}_{3}, 25{ }^{\circ} \mathrm{C}\right): \delta(\mathrm{ppm})=200.3,148.0,142.9,135.9,133.8,128.4,125.4,116.9,98.4$, 32.0, 8.4.

Elemental analysis: calcd (\%) for $\mathrm{C}_{13} \mathrm{H}_{11} \mathrm{BrO}_{2}$ (279.13): C 55.94, H 3.97; found: $\mathrm{C} 56.11$, H 4.05.

3-Bromo-2-(4-chlorophenyl)furan 5: From 1-bromo-4-chloro-benzene (96 mg, $0.5 \mathrm{mmol}$ ) and 3bromofuran (367 mg, $2.5 \mathrm{mmol}$, 5 equiv.), 5 was obtained in 39\% (50 mg) yield.

${ }^{1} \mathrm{H} \mathrm{NMR}\left(400 \mathrm{MHz}, \mathrm{CDCl}_{3}, 25^{\circ} \mathrm{C}\right): \delta(\mathrm{ppm})=7.90(\mathrm{~d}, J=8.7 \mathrm{~Hz}, 2 \mathrm{H}), 7.42(\mathrm{~d}, J=1.9 \mathrm{~Hz}, 1 \mathrm{H}), 7.39(\mathrm{~d}, J=8.7$ $\mathrm{Hz}, 2 \mathrm{H}), 6.54(\mathrm{~d}, J=1.9 \mathrm{~Hz}, 1 \mathrm{H})$.

${ }^{13} \mathrm{C}$ NMR $\left(100 \mathrm{MHz}, \mathrm{CDCl}_{3}, 25^{\circ} \mathrm{C}\right): \delta(\mathrm{ppm})=148.1,142.1,133.9,128.9,128.4,126.9,116.5,96.6$.

Elemental analysis: calcd (\%) for $\mathrm{C}_{10} \mathrm{H}_{6} \mathrm{BrClO}$ (257.51): C 46.64, H 2.35; found: C 46.36, H 2.57.

3-(3-Bromofuran-2-yl)benzonitrile 6: From 3-bromobenzonitrile ( $91 \mathrm{mg}, 0.5 \mathrm{mmol}$ ) and 3-bromofuran (367 mg, $2.5 \mathrm{mmol}, 5$ equiv.), 6 was obtained in 50\% (62 mg) yield.

${ }^{1} \mathrm{H}$ NMR $\left(400 \mathrm{MHz}, \mathrm{CDCl}_{3}, 25^{\circ} \mathrm{C}\right): \delta(\mathrm{ppm})=8.26(\mathrm{t}, J=1.3 \mathrm{~Hz}, 1 \mathrm{H}), 8.20(\mathrm{td}, J=1.3,7.7 \mathrm{~Hz}, 1 \mathrm{H}), 7.60(\mathrm{td}, J=$ $1.3,7.7 \mathrm{~Hz}, 1 \mathrm{H}), 7.53(\mathrm{t}, J=7.7 \mathrm{~Hz}, 1 \mathrm{H}), 7.47(\mathrm{~d}, J=1.9 \mathrm{~Hz}, 1 \mathrm{H}), 6.58(\mathrm{~d}, J=1.9 \mathrm{~Hz}, 1 \mathrm{H})$.

${ }^{13} \mathrm{C}$ NMR $\left(100 \mathrm{MHz}, \mathrm{CDCl}_{3}, 25{ }^{\circ} \mathrm{C}\right): \delta(\mathrm{ppm})=146.7,142.9,131.2,131.1,129.6,129.4,128.9,118.7,116.8$, 113.1, 98.2.

Elemental analysis: calcd (\%) for $\mathrm{C}_{11} \mathrm{H}_{6} \mathrm{BrNO}$ (248.08): C 53.26, H 2.44; found: C 53.41, H 2.36.

3-Bromo-2-(3-(trifluoromethyl)phenyl)furan 7: From 3-bromobenzotrifluoride (113 mg, $0.5 \mathrm{mmol}$ ) and 3-bromofuran (367 mg, $2.5 \mathrm{mmol}, 5$ equiv.), 7 was obtained in 50\% (73 mg) yield.

${ }^{1} \mathrm{H}$ NMR $\left(400 \mathrm{MHz}, \mathrm{CDCl}_{3}, 25^{\circ} \mathrm{C}\right): \delta(\mathrm{ppm})=8.22(\mathrm{~s}, 1 \mathrm{H}), 8.17(\mathrm{~d}, J=7.8 \mathrm{~Hz}, 1 \mathrm{H}), 7.63-7.51(\mathrm{~m}, 2 \mathrm{H}), 7.46$ $(\mathrm{d}, J=2.0 \mathrm{~Hz}, 1 \mathrm{H}), 6.57(\mathrm{~d}, J=2.0 \mathrm{~Hz}, 1 \mathrm{H})$.

${ }^{13} \mathrm{C}$ NMR $\left(100 \mathrm{MHz}, \mathrm{CDCl}_{3}, 25^{\circ} \mathrm{C}\right): \delta(\mathrm{ppm})=147.6,142.6,131.4,130.6,129.2,128.5,124.6(\mathrm{q}, J=3.9 \mathrm{~Hz})$, $124.1(\mathrm{q}, J=274.1 \mathrm{~Hz}), 122.3(\mathrm{q}, J=4.0 \mathrm{~Hz}), 116.7,97.5$.

${ }^{19} \mathrm{~F}$ NMR $\left(376 \mathrm{MHz}, \mathrm{CDCl}_{3}, 25^{\circ} \mathrm{C}\right): \delta(\mathrm{ppm})=-62.9(\mathrm{~s})$.

Elemental analysis: calcd (\%) for $\mathrm{C}_{11} \mathrm{H}_{6} \mathrm{BrF}_{3} \mathrm{O}$ (291.07): C 45.39, H 2.08; found: C 45.56, H 2.21.

2-(3-Bromofuran-2-yl)benzonitrile 8: From 2-bromobenzonitrile ( $91 \mathrm{mg}, 0.5 \mathrm{mmol}$ ) and 3-bromofuran (367 mg, $2.5 \mathrm{mmol}, 5$ equiv.), 8 was obtained in $42 \%$ (52 mg) yield.

${ }^{1} \mathrm{H} \mathrm{NMR}\left(400 \mathrm{MHz}, \mathrm{CDCl}_{3}, 25^{\circ} \mathrm{C}\right): \delta(\mathrm{ppm})=7.92(\mathrm{~d}, J=7.4 \mathrm{~Hz}, 1 \mathrm{H}), 7.77(\mathrm{~d}, J=7.9 \mathrm{~Hz}, 1 \mathrm{H}), 7.65(\mathrm{td}, J=1.3$, $7.9 \mathrm{~Hz}, 1 \mathrm{H}), 7.57(\mathrm{~d}, J=1.9 \mathrm{~Hz}, 1 \mathrm{H}), 7.46(\mathrm{td}, J=1.3,7.7 \mathrm{~Hz}, 1 \mathrm{H}), 6.61(\mathrm{~d}, J=1.9 \mathrm{~Hz}, 1 \mathrm{H})$.

${ }^{13} \mathrm{C}$ NMR $\left(100 \mathrm{MHz}, \mathrm{CDCl}_{3}, 2{ }^{\circ} \mathrm{C}\right): \delta(\mathrm{ppm})=146.7,143.7,134.5,132.5,132.3,129.4,128.8,118.2,116.2$, 111.0, 99.8.

Elemental analysis: calcd (\%) for $\mathrm{C}_{11} \mathrm{H}_{6} \mathrm{BrNO}$ (248.08): C 53.26, H 2.44; found: C 53.20, H 2.49.

4-(3-Bromofuran-2-yl)-3-fluorobenzaldehyde 9: From 4-bromo-2-fluorobenzaldehyde (102 mg, 0.5 mmol) and 3-bromofuran (367 mg, $2.5 \mathrm{mmol}, 5$ equiv.), 9 was obtained in $52 \%$ (70 mg) yield.

${ }_{1} \mathrm{H} \mathrm{NMR}\left(400 \mathrm{MHz}, \mathrm{CDCl}_{3}, 25^{\circ} \mathrm{C}\right): \delta(\mathrm{ppm})=10.00(\mathrm{~s}, 1 \mathrm{H}), 8.24(\mathrm{dd}, J=2.1,6.9 \mathrm{~Hz}, 1 \mathrm{H}$ ), 7.93 (ddd, $J=2.2$, 4.8, $8.5 \mathrm{~Hz}, 1 \mathrm{H}$ ), 7.53 (d, $J=1.9 \mathrm{~Hz}, 1 \mathrm{H}), 7.40-7.24(\mathrm{~m}, 1 \mathrm{H}), 6.60$ (d, $J=2.0 \mathrm{~Hz}, 1 \mathrm{H})$.

${ }^{13} \mathrm{C}$ NMR $\left(100 \mathrm{MHz}, \mathrm{CDCl}_{3}, 25^{\circ} \mathrm{C}\right): \delta(\mathrm{ppm})=190.2,163.0(\mathrm{~d}, J=263.4 \mathrm{~Hz}), 144.6(\mathrm{~d}, J=2.3 \mathrm{~Hz}), 143.8$, 132.9 (d, $J=3.1 \mathrm{~Hz}), 132.8$ (d, $J=4.6 \mathrm{~Hz}), 131.7$ (d, $J=9.9 \mathrm{~Hz}), 118.9$ (d, $J=14.7 \mathrm{~Hz}), 117.7$ (d, $J=23.0 \mathrm{~Hz}$ ), $115.8,100.2$.

${ }^{19} \mathrm{~F}$ NMR $\left(376 \mathrm{MHz}, \mathrm{CDCl}_{3}, 25^{\circ} \mathrm{C}\right): \delta(\mathrm{ppm})=-100.9(\mathrm{~s})$

Elemental analysis: calcd (\%) for $\mathrm{C}_{11} \mathrm{H}_{6} \mathrm{BrFO}_{2}$ (269.07): C 49.10, H 2.25; found: C 49.38, H 2.44. 
3-Bromo-1-(naphthalen-2-yl)furan 10: From 1-bromonaphtalene (103 mg, $0.5 \mathrm{mmol}$ ) and 3bromofuran (367 mg, $2.5 \mathrm{mmol}, 5$ equiv.), 10 was obtained in 38\% (52 mg) yield.

${ }^{1} \mathrm{H}$ NMR $\left(400 \mathrm{MHz}, \mathrm{CDCl}_{3}, 25^{\circ} \mathrm{C}\right): \delta(\mathrm{ppm})=7.98-7.90(\mathrm{~m}, 3 \mathrm{H}), 7.73(\mathrm{dd}, J=1.3,7.1 \mathrm{~Hz}, 1 \mathrm{H}), 7.61(\mathrm{~d}, J=$ $2.0 \mathrm{~Hz}, 1 \mathrm{H}), 7.59-7.53(\mathrm{~m}, 3 \mathrm{H}), 6.68(\mathrm{~d}, J=2.0 \mathrm{~Hz}, 1 \mathrm{H})$.

${ }^{13} \mathrm{C}$ NMR $\left(100 \mathrm{MHz}, \mathrm{CDCl}_{3}, 25^{\circ} \mathrm{C}\right): \delta(\mathrm{ppm})=150.5,142.8,133.9,131.7,130.0,129.0,128.5,126.8,126.3$, 126.1, 125.1, 115.3, 99.1.

Elemental analysis: calcd (\%) for $\mathrm{C}_{14} \mathrm{H}_{9} \mathrm{BrO}$ (273.13): C 61.57, H 3.32; found: C 61.46, H 3.28.

3-Bromo-2-(naphthalen-2-yl)furan 11: From 2-bromonaphtalene (103 mg, $0.5 \mathrm{mmol}$ ) and 3bromofuran (367 mg, $2.5 \mathrm{mmol}$, 5 equiv.), 11 was obtained in 45\% (61 mg) yield.

${ }^{1} \mathrm{H} \mathrm{NMR}\left(400 \mathrm{MHz}, \mathrm{CDCl}_{3}, 25^{\circ} \mathrm{C}\right): \delta(\mathrm{ppm})=8.45(\mathrm{~s}, 1 \mathrm{H}), 8.08(\mathrm{dd}, J=1.8,8.7 \mathrm{~Hz}, 1 \mathrm{H}), 7.94-7.86(\mathrm{~m}, 2 \mathrm{H})$, $7.86-7.81(\mathrm{~m}, 1 \mathrm{H}), 7.54-7.45(\mathrm{~m}, 3 \mathrm{H}), 6.59(\mathrm{~d}, J=2.0 \mathrm{~Hz}, 1 \mathrm{H})$.

${ }^{13} \mathrm{C}$ NMR $\left(100 \mathrm{MHz}, \mathrm{CDCl}_{3}, 2{ }^{\circ} \mathrm{C}\right): \delta(\mathrm{ppm})=149.2,142.1,133.3,132.9,128.6,128.3,127.8,127.3,126.6$, $126.6,124.9,123.4,116.6,96.6$.

Elemental analysis: calcd (\%) for $\mathrm{C}_{14} \mathrm{H}_{9} \mathrm{BrO}$ (273.13): C 61.57, H 3.32; found: C 61.79, H 3.51.

3-(3-Bromofuran-2-yl)pyridine 12: From 3-bromopyridine (79 $\mathrm{mg}, 0.5 \mathrm{mmol}$ ) and 3-bromofuran (367 mg, $2.5 \mathrm{mmol}$, 5 equiv.), 12 was obtained in $45 \%$ (50 mg) yield.

${ }^{1} \mathrm{H} \mathrm{NMR}\left(400 \mathrm{MHz}, \mathrm{CDCl}_{3}, 25^{\circ} \mathrm{C}\right): \delta(\mathrm{ppm})=9.23(\mathrm{brs}, 1 \mathrm{H}), 8.57(\mathrm{brs}, 1 \mathrm{H}), 8.38-8.11(\mathrm{~m}, 1 \mathrm{H}), 7.47(\mathrm{~d}, J=$ $2.0 \mathrm{~Hz}, 1 \mathrm{H}), 7.36(\mathrm{dd}, J=4.8,8.2 \mathrm{~Hz}, 1 \mathrm{H}), 6.57(\mathrm{~d}, J=2.0 \mathrm{~Hz}, 1 \mathrm{H})$.

${ }^{13} \mathrm{C}$ NMR $\left(100 \mathrm{MHz}, \mathrm{CDCl}_{3}, 25^{\circ} \mathrm{C}\right): \delta(\mathrm{ppm})=148.8,146.8,146.5,142.9,132.5,123.5,116.6,97.8$.

Elemental analysis: calcd (\%) for $\mathrm{C}_{9} \mathrm{H}_{6} \mathrm{BrNO}$ (224.06): C 48.25, H 2.70; found: C 48.21, H 2.83.

5-(3-Bromofuran-2-yl)pyrimidine 13: From 5-bromopyrimidine (79 mg, $0.5 \mathrm{mmol}$ ) and 3-bromofuran (367 mg, $2.5 \mathrm{mmol}, 5$ equiv.), 13 was obtained in 52\% (58 mg) yield.

${ }^{1} \mathrm{H} \mathrm{NMR}\left(400 \mathrm{MHz}, \mathrm{CDCl}_{3}, 25^{\circ} \mathrm{C}\right): \delta(\mathrm{ppm})=9.34(\mathrm{~s}, 2 \mathrm{H}), 9.18(\mathrm{~s}, 1 \mathrm{H}), 7.53(\mathrm{~d}, J=2.0 \mathrm{~Hz}, 1 \mathrm{H}), 6.62(\mathrm{~d}, J=$ $2.0 \mathrm{~Hz}, 1 \mathrm{H})$.

${ }^{13} \mathrm{C}$ NMR $\left(100 \mathrm{MHz}, \mathrm{CDCl}_{3}, 25^{\circ} \mathrm{C}\right): \delta(\mathrm{ppm})=157.3,153.0,144.0,143.9,124.6,116.8,99.5$.

Elemental analysis: calcd (\%) for $\mathrm{C}_{8} \mathrm{H}_{5} \mathrm{BrN}_{2} \mathrm{O}$ (225.05): C 42.70, H 2.24; found: C 42.96, H 2.37.

3-(3-Bromofuran-2-yl)quinoline 14: From 3-bromoquinoline (104 mg, $0.5 \mathrm{mmol}$ ) and 3-bromofuran (367 mg, $2.5 \mathrm{mmol}$, 5 equiv.), 14 was obtained in 50\% (69 mg) yield.

${ }^{1} \mathrm{H} \mathrm{NMR}\left(400 \mathrm{MHz}, \mathrm{CDCl}_{3}, 25^{\circ} \mathrm{C}\right): \delta(\mathrm{ppm})=9.50(\mathrm{~d}, J=2.3 \mathrm{~Hz}, 1 \mathrm{H}), 8.69(\mathrm{~d}, J=2.3 \mathrm{~Hz}, 1 \mathrm{H}), 8.11(\mathrm{~d}, J=8.4$ $\mathrm{Hz}, 1 \mathrm{H}), 7.87(\mathrm{~d}, J=8.1 \mathrm{~Hz}, 1 \mathrm{H}), 7.73(\mathrm{t}, J=6.8 \mathrm{~Hz}, 1 \mathrm{H}), 7.57(\mathrm{t}, J=7.9 \mathrm{~Hz}, 1 \mathrm{H}), 7.52$ (d, J = 2.0 Hz, 1H), 6.61 $(\mathrm{d}, J=2.0 \mathrm{~Hz}, 1 \mathrm{H})$.

${ }^{13} \mathrm{C}$ NMR $\left(100 \mathrm{MHz}, \mathrm{CDCl}_{3}, 2{ }^{\circ} \mathrm{C}\right): \delta(\mathrm{ppm})=147.7,147.2,146.9,143.0,131.8,130.0,129.4,128.3,127.6$, 127.4, 123.3, 116.7, 98.0.

Elemental analysis: calcd (\%) for $\mathrm{C}_{13} \mathrm{H}_{8} \mathrm{BrNO}$ (274.12): C 56.96, H 2.94; found: C 57.08, H 2.69.

General procedure for the $\mathbf{C 2 , C 5}$-diarylation reaction of 3-bromofuran: As a typical experiment, the reaction of the aryl bromide ( $1 \mathrm{mmol}), 3$-bromofuran $(0.5 \mathrm{mmol}, 1$ equiv., $73 \mathrm{mg}$ ) and KOAc $(1.5 \mathrm{mmol}, 3$ equiv., $147 \mathrm{mg}$ ) at $120^{\circ} \mathrm{C}$ during $8 \mathrm{~h}$ in DMA (2 mL) in the presence of $\mathrm{Pd}(\mathrm{OAc})_{2}$ (2 mol\%, $0.01 \mathrm{mmol}, 2.24$ $\mathrm{mg}$ ) (see tables or schemes) under argon affords the diarylation product after evaporation of the solvent and purification on silica gel.

3-Bromo-2,5-bis(4-(trifluoromethyl)phenyl)furan 16: From 4-bromobenzotrifluoride (223 mg, 1 mmol, 2 equiv) and 3-bromofuran (73 mg, $0.5 \mathrm{mmol}, 1$ equiv.), 16 was obtained in 56\% (122 mg) yield. 
${ }^{1} \mathrm{H}$ NMR (400 MHz, $\left.\mathrm{CDCl}_{3}, 25^{\circ} \mathrm{C}\right): \delta(\mathrm{ppm}) 8.17(\mathrm{~d}, J=8.8 \mathrm{~Hz}, 2 \mathrm{H}), 7.80(\mathrm{~d}, J=8.3 \mathrm{~Hz}, 2 \mathrm{H}), 7.75-7.66(\mathrm{~m}$, $4 \mathrm{H}), 6.93(\mathrm{~s}, 1 \mathrm{H})$.

${ }^{13} \mathrm{C}$ NMR $\left(100 \mathrm{MHz}, \mathrm{CDCl}_{3}, 25^{\circ} \mathrm{C}\right): \delta(\mathrm{ppm})=152.0,147.7,132.5,132.4,130.2(\mathrm{q}, J=29.3 \mathrm{~Hz}), 129.9(\mathrm{q}, J=$ $28.4 \mathrm{~Hz}), 126.0(\mathrm{q}, J=3.9 \mathrm{~Hz}), 125.6(\mathrm{q}, J=3.9 \mathrm{~Hz}), 125.5,124.1,124.0(\mathrm{q}, J=273.5 \mathrm{H}), 123.9(\mathrm{q}, J=273.5$ H), 113.4, 100.0.

${ }^{19} \mathrm{~F} \mathrm{NMR}\left(376 \mathrm{MHz}, \mathrm{CDCl}_{3}, 25^{\circ} \mathrm{C}\right): \delta(\mathrm{ppm})=-62.7(2)$.

Elemental analysis: calcd (\%) for $\mathrm{C}_{18} \mathrm{H}_{9} \mathrm{BrF}_{6} \mathrm{O}$ (435.16): C 49.68, H 2.08; found: $\mathrm{C} 49.67, \mathrm{H} 2.00$.

3-Bromo-2,5-bis(3-(trifluoromethyl)phenyl)furan 17: From 3-bromobenzotrifluoride (223 mg, 1 mmol, 2 equiv) and 3-bromofuran (73 mg, $0.5 \mathrm{mmol}, 1$ equiv.), 17 was obtained in 53\% (115 mg) yield.

${ }^{1} \mathrm{H} \mathrm{NMR}\left(400 \mathrm{MHz}, \mathrm{CDCl}_{3}, 25^{\circ} \mathrm{C}\right): \delta(\mathrm{ppm})=8.30(\mathrm{~s}, 1 \mathrm{H}), 8.26-8.19(\mathrm{~m}, 1 \mathrm{H}), 7.92(\mathrm{~s}, 1 \mathrm{H}), 7.87(\mathrm{dt}, J=2.0$, $5.8 \mathrm{~Hz}, 1 \mathrm{H}), 7.66-7.52(\mathrm{~m}, 4 \mathrm{H}), 6.90(\mathrm{~s}, 1 \mathrm{H})$.

${ }^{13} \mathrm{C}$ NMR $\left(100 \mathrm{MHz}, \mathrm{CDCl}_{3}, 25^{\circ} \mathrm{C}\right): \delta(\mathrm{ppm})=152.0,147.6,131.7(\mathrm{q}, J=25.4 \mathrm{~Hz}), 131.3(\mathrm{~d}, J=25.3 \mathrm{~Hz})$, $130.2,130.2,129.7,129.3,128.6(\mathrm{~m}), 127.2,(\mathrm{~m}), 125.1$ (q, $J=3.8 \mathrm{~Hz}), 124.9$ (q, $J=3.8 \mathrm{~Hz}), 124.1(\mathrm{q}, J=$ $273.2 \mathrm{~Hz}), 124.0(\mathrm{q}, J=273.2 \mathrm{~Hz}), 122.3(\mathrm{q}, J=4.0 \mathrm{~Hz}), 120.9$ (d, $J=3.9 \mathrm{~Hz}), 112.9$, 99.6.

${ }^{19} \mathrm{~F}$ NMR $\left(376 \mathrm{MHz}, \mathrm{CDCl}_{3}, 25^{\circ} \mathrm{C}\right): \delta(\mathrm{ppm})=-62.9(\mathrm{~d})$.

Elemental analysis: calcd (\%) for $\mathrm{C}_{18} \mathrm{H}_{9} \mathrm{BrF}_{6} \mathrm{O}$ (435.16): C 49.68, H 2.08; found: C $49.89 \mathrm{H} 1.97$.

3-Bromo-2,5-bis(2-(trifluoromethyl)phenyl)furan 18: From 2-bromobenzotrifluoride (223 mg, 1 mmol, 2 equiv) and 3-bromofuran (73 mg, $0.5 \mathrm{mmol}, 1$ equiv.), 18 was obtained in 47\% (102 mg) yield.

${ }^{1} \mathrm{H} \mathrm{NMR}\left(400 \mathrm{MHz}, \mathrm{CDCl}_{3}, 25^{\circ} \mathrm{C}\right): \delta(\mathrm{ppm})=7.89-7.81(\mathrm{~m}, 2 \mathrm{H}), 7.80-7.73(\mathrm{~m}, 2 \mathrm{H}), 7.70-7.53(\mathrm{~m}, 3 \mathrm{H})$, $7.50-7.41(\mathrm{~m}, 1 \mathrm{H}), 6.93(\mathrm{~s}, 1 \mathrm{H})$.

${ }^{13} \mathrm{C}$ NMR $\left(100 \mathrm{MHz}, \mathrm{CDCl}_{3}, 2{ }^{\circ} \mathrm{C}\right): \delta(\mathrm{ppm})=150.4,148.2,132.5,132.1,131.7,130.0,129.7,129.6(\mathrm{q}, J=$ $31.5 \mathrm{~Hz}), 128.6,127.1(\mathrm{q}, J=5.4 \mathrm{~Hz}), 126.7(\mathrm{q}, J=5.9 \mathrm{~Hz}), 126.7(\mathrm{q}, J=31.2 \mathrm{~Hz}), 123.9$ (q, $J=271.6 \mathrm{~Hz})$, $123.8(\mathrm{q}, J=271.6 \mathrm{~Hz}), 115.2(\mathrm{q}, J=4.7 \mathrm{~Hz}), 100.5$.

${ }^{19} \mathrm{~F}$ NMR $\left(376 \mathrm{MHz}, \mathrm{CDCl}_{3}, 25^{\circ} \mathrm{C}\right): \delta(\mathrm{ppm})=-59.6,-60.0$.

Elemental analysis: calcd (\%) for $\mathrm{C}_{18} \mathrm{H}_{9} \mathrm{BrF}_{6} \mathrm{O}$ (435.16): C 49.68, H 2.08; found: $\mathrm{C} 49.58 \mathrm{H} 2.34$.

6,6'-(3-Bromofuran-2,5-diyl)bis(2-(trifluoromethyl)pyridine) 19: From 2-bromo-6trifluoromethylpyridine (192 mg, $1 \mathrm{mmol}, 2$ equiv.) and 3-bromofuran (73 mg, $0.5 \mathrm{mmol}, 1$ equiv.), 19 was obtained in 49\% (107 mg) yield.

${ }^{1} \mathrm{H}$ NMR $\left(300 \mathrm{MHz}, \mathrm{CDCl}_{3}, 25{ }^{\circ} \mathrm{C}\right): \delta(\mathrm{ppm})=8.19(\mathrm{~d}, J=8.1 \mathrm{~Hz}, 1 \mathrm{H}), 8.05-7.93(\mathrm{~m}, 3 \mathrm{H}), 7.67-7.59(\mathrm{~m}$, 2H), $7.44(\mathrm{~s}, 1 \mathrm{H})$.

${ }^{13} \mathrm{C}$ NMR $\left(100 \mathrm{MHz}, \mathrm{CDCl}_{3}, 25^{\circ} \mathrm{C}\right): \delta(\mathrm{ppm})=152.6,148.5,148.5(\mathrm{q}, J=25.3 \mathrm{~Hz}), 148.2,148.2(\mathrm{q}, J=25.9$ $\mathrm{Hz}), 147.6,138.3,138.0,122.8,121.3,121.3$ (q, $J=274.5 \mathrm{~Hz}), 121.2$ (q, $J=274.5 \mathrm{~Hz}), 119.3$ (t, $J=2.9 \mathrm{~Hz}$ ), $119.0(\mathrm{q}, J=2.7 \mathrm{~Hz}), 116.9,103.3$.

${ }^{19} \mathrm{~F}$ NMR $\left(376 \mathrm{MHz}, \mathrm{CDCl}_{3}, 25^{\circ} \mathrm{C}\right): \delta(\mathrm{ppm})=-68.2,-68.3$.

Elemental analysis: calcd (\%) for $\mathrm{C}_{16} \mathrm{H}_{7} \mathrm{BrF}_{6} \mathrm{~N}_{2} \mathrm{O}$ (437.14): C 43.96, $\mathrm{H} 1.61$; found: $\mathrm{C} 44.09 \mathrm{H} 1.98$.

2,3,5-Tris(3,5-bis(trifluoromethyl)phenyl)-4-bromofuran 20: the reaction of 1-bromo-3,5bis(trifluoromethyl)benzene ( $586 \mathrm{mg}, 2 \mathrm{mmol}, 4$ equiv.), 3-bromofuran (73 mg, $0.5 \mathrm{mmol}, 1 \mathrm{equiv}$.) and KOAc (180 mg, $3 \mathrm{mmol}, 4$ equiv.) at $120^{\circ} \mathrm{C}$ during $8 \mathrm{~h}$ in DMA $(2 \mathrm{~mL})$ in the presence of $\mathrm{Pd}(\mathrm{OAc})_{2}(2.24$ $\mathrm{mg}, 0.01 \mathrm{mmol}, 2 \mathrm{~mol} \%)$ under argon affords, after evaporation of the solvent and purification, product 20 in 61\% (239 mg) yield. 
${ }^{1} \mathrm{H}$ NMR $\left(300 \mathrm{MHz}, \mathrm{CDCl}_{3}, 25^{\circ} \mathrm{C}\right): \delta(\mathrm{ppm})=8.52(\mathrm{~s}, 2 \mathrm{H}), 8.05(\mathrm{~s}, 1 \mathrm{H}), 7.95(\mathrm{~s}, 1 \mathrm{H}), 7.89(\mathrm{~s}, 2 \mathrm{H}), 7.84(\mathrm{~s}$, $1 \mathrm{H}), 7.82(\mathrm{~s}, 2 \mathrm{H})$.

${ }^{13} \mathrm{C}$ NMR $\left(100 \mathrm{MHz}, \mathrm{CDCl}_{3}, 25^{\circ} \mathrm{C}\right): \delta(\mathrm{ppm})=147.6,147.5,133.2(\mathrm{q}, J=34.7 \mathrm{~Hz}), 132.9(\mathrm{q}, J=35.1 \mathrm{~Hz})$, 132.8 (q, $J=35.2 \mathrm{~Hz}), 132.6,130.5,130.5(\mathrm{~m}), 130.4,126.0(\mathrm{~m}), 125.8(\mathrm{~m}) 125.2,123.2$ (q, $J=273.1 \mathrm{~Hz})$, $123.2(\mathrm{~m}), 123.0(\mathrm{q}, J=273.1 \mathrm{~Hz}), 122.8(\mathrm{q}, J=3.8 \mathrm{~Hz}), 122.8(\mathrm{q}, J=273.1 \mathrm{~Hz}), 103.0$.

${ }^{19} \mathrm{~F}$ NMR $\left(376 \mathrm{MHz}, \mathrm{CDCl}_{3}, 25^{\circ} \mathrm{C}\right): \delta(\mathrm{ppm})=-63.1(2),-63.5$.

Elemental analysis: calcd (\%) for $\mathrm{C}_{28} \mathrm{H}_{9} \mathrm{BrF}_{18} \mathrm{O}$ (783.25): C 42.94, H 1.16; found: C $42.75 \mathrm{H} \mathrm{1.01.}$

2-(Naphthalen-1-yl)-3-phenylfuran 22: The reaction of 3-bromo-2-(naphthalen-1-yl)furan (10) (271 $\mathrm{mg}, 1 \mathrm{mmol})$, phenylboronic acid (180 mg, $1.5 \mathrm{mmol})$ and $\mathrm{K}_{3} \mathrm{PO}_{4}(424 \mathrm{mg}, 2 \mathrm{mmol})$ at $80{ }^{\circ} \mathrm{C}$ over $16 \mathrm{~h}$ in 1,4-dioxane (4 mL) in the presence of $\operatorname{PdCl}\left(\mathrm{C}_{3} \mathrm{H}_{5}\right)(\mathrm{dppb})(12 \mathrm{mg}, 0.02 \mathrm{mmol})$ under argon affords, after evaporation of the solvent and purification on silica gel, product 22 in 78\% (211 mg) yield.

${ }^{1} \mathrm{H}$ NMR $\left(300 \mathrm{MHz}, \mathrm{CDCl}_{3}, 25^{\circ} \mathrm{C}\right): \delta(\mathrm{ppm})=7.94-7.88(\mathrm{~m}, 2 \mathrm{H}), 7.84(\mathrm{~d}, J=8.4 \mathrm{~Hz}, 1 \mathrm{H}), 7.64(\mathrm{~d}, J=1.9 \mathrm{~Hz}$, 1H), $7.54-7.45(\mathrm{~m}, 2 \mathrm{H}), 7.45-7.37(\mathrm{~m}, 2 \mathrm{H}), 7.24-7.18(\mathrm{~m}, 2 \mathrm{H}), 7.18-7.12(\mathrm{~m}, 3 \mathrm{H}), 6.82$ (d, J = $1.9 \mathrm{~Hz}$, $1 \mathrm{H})$.

${ }^{13} \mathrm{C}$ NMR $\left(100 \mathrm{MHz}, \mathrm{CDCl}_{3}, 25{ }^{\circ} \mathrm{C}\right): \delta(\mathrm{ppm})=148.5,142.4,133.9,133.4,132.0,129.3,129.1,128.9,128.4$, $128.3,127.6,126.6,126.5,126.1,125.3,123.8,111.7$.

Elemental analysis: calcd (\%) for $\mathrm{C}_{20} \mathrm{H}_{14} \mathrm{O}$ (270.33): C 88.86, H 5.22; found: C 88.96 H 5.35.

General procedure for the C5-arylation reaction of 2-(naphthalen-1-yl)-3-phenylfuran (22): As a typical experiment, the reaction of the aryl bromide $(0.75 \mathrm{mmol}), 2$-(naphthalen-1-yl)-3-phenylfuran (22) ( 0.5 mmol, 1 equiv) and KOAc ( 1 mmol, 2 equiv., $98 \mathrm{mg})$ at $120{ }^{\circ} \mathrm{C}$ during $8 \mathrm{~h}$ in DMA $(2 \mathrm{~mL})$ in the presence of $\mathrm{PdCl}\left(\mathrm{C}_{3} \mathrm{H}_{5}\right)(\mathrm{dppb})(12 \mathrm{mg}, 0.02 \mathrm{mmol}$ ) (see tables or schemes) under argon affords the arylation product after evaporation of the solvent and purification on silica gel.

4-(5-(Naphthalen-1-yl)-4-phenylfuran-2-yl)benzonitrile 23: From 4-bromobenzonitrile (137 mg, $0.75 \mathrm{mmol}, 1.5$ equiv.) and 2-(naphthalen-1-yl)-3-phenylfuran (22) (135 mg, $0.5 \mathrm{mmol}, 1$ equiv), 23 was obtained in $71 \%$ (132 $\mathrm{mg}$ ) yield.

${ }_{1}^{1} \mathrm{H} \mathrm{NMR}\left(400 \mathrm{MHz}, \mathrm{CDCl}_{3}, 25^{\circ} \mathrm{C}\right): \delta(\mathrm{ppm})=8.00-7.92(\mathrm{~m}, 3 \mathrm{H}), 7.86(\mathrm{~d}, J=8.5 \mathrm{~Hz}, 2 \mathrm{H}), 7.70(\mathrm{~d}, J=8.5 \mathrm{~Hz}$, $2 \mathrm{H}$ ), 7.59 (ddd, $J=1.3,7.6,8.1 \mathrm{~Hz}, 1 \mathrm{H}), 7.56-7.50(\mathrm{~m}, 2 \mathrm{H}), 7.50-7.42(\mathrm{~m}, 1 \mathrm{H}), 7.32-7.20(\mathrm{~m}, 6 \mathrm{H})$.

${ }^{13} \mathrm{C}$ NMR $\left(100 \mathrm{MHz}, \mathrm{CDCl}_{3}, 2{ }^{\circ} \mathrm{C}\right): \delta(\mathrm{ppm})=151.6,149.9,134.5,134.1,132.8,132.8,131.8,130.0,129.2$, 128.7, 128.6, 128.4, 127.7, 127.3, 126.9, 126.6, 126.4, 126.0, 125.4, 124.1, 119.1, 110.5, 110.3.

Elemental analysis: calcd (\%) for $\mathrm{C}_{27} \mathrm{H}_{17} \mathrm{NO}$ (371.44): C 87.31, H 4.61; found: C 87.45, H 4.81.

3-(5-(naphthalen-1-yl)-4-phenylfuran-2-yl)quinoline 24: From 3-bromoquinoline (156 mg, 0.75 mmol, 1.5 equiv.) and 2-(naphthalen-1-yl)-3-phenylfuran (22) (135 mg, $0.5 \mathrm{mmol}, 1$ equiv), 24 was obtained in $81 \%$ (161 mg) yield.

${ }^{1} \mathrm{H} \mathrm{NMR}\left(400 \mathrm{MHz}, \mathrm{CDCl}_{3}, 25^{\circ} \mathrm{C}\right): \delta(\mathrm{ppm})=9.38(\mathrm{~d}, J=2.1 \mathrm{~Hz}, 1 \mathrm{H}), 8.48(\mathrm{~s}, 1 \mathrm{H}), 8.17(\mathrm{~d}, J=9.1 \mathrm{~Hz}, 1 \mathrm{H})$, $8.04(\mathrm{~d}, J=8.5 \mathrm{~Hz}, 1 \mathrm{H}), 7.97(\mathrm{t}, J=7.1 \mathrm{~Hz}, 2 \mathrm{H}), 7.85(\mathrm{~d}, J=8.2 \mathrm{~Hz}, 1 \mathrm{H}), 7.77-7.64(\mathrm{~m}, 2 \mathrm{H}), 7.58-7.51(\mathrm{~m}$, $4 \mathrm{H}), 7.35(\mathrm{~d}, J=7.5 \mathrm{~Hz}, 3 \mathrm{H}), 7.31-7.20(\mathrm{~m}, 3 \mathrm{H})$.

${ }^{13} \mathrm{C}$ NMR $\left(100 \mathrm{MHz}, \mathrm{CDCl}_{3}, 25^{\circ} \mathrm{C}\right): \delta(\mathrm{ppm})=150.9,149.2,146.9,146.9,134.0,132.8,131.8,129.7,129.5$, $129.3,129.2,129.1,128.6,128.5,128.4,128.0,128.0,127.6,127.4,127.4,127.1,126.7,126.2,126.0$, $125.4,123.9,108.8$.

Elemental analysis: calcd (\%) for $\mathrm{C}_{29} \mathrm{H}_{19} \mathrm{NO}$ (397.48): C 87.63, H 4.82; found: C 87.89, H 5.01.

\section{Funding Information}


A.S. acknowledges the Indo-French Centre for the Promotion of Advanced Research (IFCPAR, № 5705-1) for a PhD grant and research support.

\section{Acknowledgment}

We thank CNRS and "Rennes Metropole" for providing financial support.

\section{Supporting Information}

Supporting information contains the ${ }^{1} \mathrm{H}$ and ${ }^{13} \mathrm{C}$ charts of all compounds.

\section{References}

1. Raczko, J.; Jurczak, J., Furan in the synthesis of natural products. In Studies in Natural Products Chemistry, Atta ur, R., Ed. Elsevier: 1995; Vol. 16, pp 639-685.

2. (a) Sperry, J. B.; Wright, D. L. Curr. Opin. Drug Discov. Devel. 2005, 8, 723-740; (b) Taylor, R. D.; MacCoss, M.; Lawson, A. D. G. J. Med. Chem. 2014, 57, 5845-5859; (c) Lukevits, É.; Demicheva, L. Chem. Heterocycl. Compd. 1993, 29, 243-267.

3. Wang, G. Q.; Jiang, M.; Zhang, Q.; Wang, R.; Qu, X. L.; Zhou, G. Y. Prog. Chem. 2018, 30, 719-736.

4. (a) Li, B. L. Chin. J. Org. Chem. 2015, 35, 2487-2506; (b) Huang, P. S.; Du, J.; Biewer, M. C.; Stefan, M. C. J. Mater. Chem. A 2015, 3, 6244-6257.

5. (a) Sousa, A. F.; Vilela, C.; Fonseca, A. C.; Matos, M.; Freire, C. S. R.; Gruter, G.-J. M.; Coelho, J. F. J.; Silvestre, A. J. D. Polym. Chem. 2015, 6, 5961-5983; (b) Makarov, A. S.; Uchuskin, M. G.; Trushkov, I. V. Synthesis 2018, 50, 3059-3086; (c) Yin, Z.; He, Y.; Chiu, P. Chem. Soc. Rev. 2018, 47, 8881-8924.

6. $\quad$ Moran, W. J.; Rodriguez, A. Org. Prep. Proced. Int. 2012, 44, 103-130.

7. (a) For general reviews on C-H bond functionalizations, see: Kakiuchi, F.; Kochi, T. Synthesis 2008, 3013-3039; (b) Ackermann, L.; Vicente, R.; Kapdi, A. R. Angew. Chem. Int. Ed. 2009, 48, 9792-9826; (c) Bellina, F.; Rossi, R. Tetrahedron 2009, 65, 10269-10310; (d) Lyons, T. W.; Sanford, M. S. Chem. Rev. 2010, 110, 1147-1169; (e) Beck, E. M.; Gaunt, M. J. Top. Curr. Chem. 2010, 292, 85-121; (f) Satoh, T.; Miura, M. Synthesis 2010, 3395-3409; (g) Cho, S. H.; Kim, J. Y.; Kwak, J.; Chang, S. Chem. Soc. Rev. 2011, 40, 50685083; (h) Li, B.-J.; Shi, Z.-J. Chem. Soc. Rev. 2012, 41, 5588-5598; (i) Kozhushkov, S. I.; Ackermann, L. Chem. Sci. 2013, 4, 886-896; (j) Rossi, R.; Bellina, F.; Lessi, M.; Manzini, C. Adv. Synth. Catal. 2014, 356, 17-117; (k) Zhang, M.; Zhang, Y.; Jie, X.; Zhao, H.; Li, G.; Su, W. Org. Chem. Front. 2014, 1, 843-895; (l) Yadav, M. R.; Rit, R. K.; Shankar, M.; Sahoo, A. K. Asian J. Org. Chem. 2015, 4, 846-864; (m) Hirano, K.; Miura, M. Chem. Lett. 2015, 44, 878-873; (n) Mao, S.; Li, H.; Shi, X.; Soulé, J.-F.; Doucet, H. ChemCatChem 2019, 11, $269-$ 286; (o) Murai, M.; Takai, K. Synthesis 2019, 51, 40-54.

8. Ohta, A.; Akita, Y.; Ohkuwa, T.; Chiba, M.; Fukunaga, R.; Miyafuji, A.; Nakata, T.; Tani, N.; Aoyagi, Y. Heterocycles 1991, 31, 1951-1958.

9. Fu, H. Y.; Doucet, H. Eur. J. Org. Chem. 2011, 7163-7173.

10. Glover, B.; Harvey, K. A.; Liu, B.; Sharp, M. J.; Tymoschenko, M. F. Org. Lett. 2003, 5, 301-304.

11. Dong, J. J.; Roy, D.; Roy, R. J.; Ionita, M.; Doucet, H. Synthesis 2011, 3530-3546.

12. Battace, A.; Lemhadri, M.; Zair, T.; Doucet, H.; Santelli, M. Organometallics 2007, 26, 472-474.

13. (a) Kobayashi, K.; Sugie, A.; Takahashi, M.; Masui, K.; Mori, A. Org. Lett. 2005, 7, 5083-5085; (b) René, O.; Fagnou, K. Org. Lett. 2010, 12, 2116-2119; (c) Brahim, M.; Smari, I.; Ben Ammar, H.; Ben Hassine, B.; Soule, J.-F.; Doucet, H. Org. Chem. Front. 2015, 2, 917-926.

14. CCDC 1883207 (1) contains the supplementary crystallographic data for this paper.

15. (a) For the synthesis of 2-aryl-3-bromofurans via cyclization reactions, see: Obrecht, D. Helv. Chim. Acta 1989, 72, 447-456; (b) For the synthesis of 2-aryl-3-bromofurans using photoredox system, see: 
Maity, P.; Kundu, D.; Ranu, B. C. Eur. J. Org. Chem. 2015, 2015, 1727-1734; (c) For the synthesis of 2-aryl3-bromofurans via Suzuki reactions, see :Liu, J.-t.; Simmons, C. J.; Xie, H.; Yang, F.; Zhao, X.-l.; Tang, Y.; Tang, W. Adv. Synth. Catal. 2017, 359, 693-697.

16. (a) Shibahara, F.; Yamaguchi, E.; Murai, T. Chem. Commun. 2010, 46, 2471-2473; (b) Okazawa, T.; Satoh, T.; Miura, M.; Nomura, M. J. Am. Chem. Soc. 2002, 124, 5286-5287.

17. (a) For selected examples of synthesis of 2,3,5-triaryl furans containing three different aryl groups, see: Dudnik, A. S.; Gevorgyan, V. Angew. Chem. Int. Ed. 2007, 46, 5195-5197; (b) Mothe, S. R.; Lauw, S. J. L.; Kothandaraman, P.; Chan, P. W. H. J. Org. Chem. 2012, 77, 6937-6947; (c) Wu, J.; Yoshikai, N. Angew. Chem. Int. Ed. 2015, 54, 11107-11111; (d) Wu, Y.; Huang, Z.; Luo, Y.; Liu, D.; Deng, Y.; Yi, H.; Lee, J.F.; Pao, C.-W.; Chen, J.-L.; Lei, A. Org. Lett. 2017, 19, 2330-2333. 